ARTICLE

\title{
Rational flux-tuning of Halomonas bluephagenesis for co-production of bioplastic PHB and ectoine
}

Hong Ma ${ }^{1,4}$, Yiqing Zhao ${ }^{1,4}$, Wuzhe Huang ${ }^{1,2}$, Lizhan Zhang $\mathbb{B}^{1}$, Fuqing $\mathrm{Wu}^{1}$, Jianwen Ye ${ }^{1,3 凶} \&$ Guo-Qiang Chen (1) 1,2,3凶

Ectoine, a compatible solute synthesized by many halophiles for hypersalinity resistance, has been successfully produced by metabolically engineered Halomonas bluephagenesis, which is a bioplastic poly(3-hydroxybutyrate) producer allowing open unsterile and continuous conditions. Here we report a de novo synthesis pathway for ectoine constructed into the chromosome of $H$. bluephagenesis utilizing two inducible systems, which serve to fine-tune the transcription levels of three clusters related to ectoine synthesis, including ect $A B C$, lys $C$ and asd based on a GFP-mediated transcriptional tuning approach. Combined with bypasses deletion, the resulting recombinant $H$. bluephagenesis TD-ADEL-58 is able to produce $28 \mathrm{~g} \mathrm{~L}^{-1}$ ectoine during a $28 \mathrm{~h}$ fed-batch growth process. Co-production of ectoine and PHB is achieved to $8 \mathrm{~g} \mathrm{~L}^{-1}$ ectoine and $32 \mathrm{~g} \mathrm{~L}^{-1}$ dry cell mass containing $75 \%$ PHB after a $44 \mathrm{~h}$ growth. $H$. bluephagenesis demonstrates to be a suitable co-production chassis for polyhydroxyalkanoates and non-polymer chemicals such as ectoine.

\footnotetext{
${ }^{1}$ Center for Synthetic and Systems Biology, School of Life Sciences, Tsinghua University, Beijing 100084, China. ${ }^{2}$ MOE Key Lab of Industrial Biocatalysis, Department of Chemical Engineering, Tsinghua University, Beijing 100084, China. ${ }^{3}$ Tsinghua-Peking Center for Life Sciences, School of Life Sciences, Tsinghua University, Beijing 100084, China. ${ }^{4}$ These authors contributed equally: Hong Ma, Yiqing Zhao. ${ }^{凶}$ email: yejianwen@phalab.org; chenga@mail.tsinghua.edu.cn
} 
Ectoine (1,4,5,6-tetrahydro-2-methyl-4-pyrimidinecarboxylic acid) is one of the best well-known compatible solutes produced by halophiles for hypersalinity resistance ${ }^{1}$. Ectoine was reported to show protections for living cells by enhancing the stabilities of proteins, nucleic acids, and cell membranes, enabling survival of halophiles under the circumstances of hypersalinity, high temperatures, extreme $\mathrm{pH}$ and exposure to chemical agents ${ }^{2-7}$. Therefore, ectoine has been exploited for commercial applications as an ingredient for cosmetics $^{8}$, medicines ${ }^{9}$, and organ transplantation maintenance ${ }^{10}$. However, the high production cost is still unacceptable for wider applications ${ }^{11}$.

Commercially, fermentative production of ectoine by microorganisms under sterile bioprocesses is the major solution to meet its increasing demands ${ }^{12}$. The major biosynthesis pathway of ectoine containing its operon ect $A B C$ encoding L-2,4-diaminobutyrate acetyltransferase (ectA), L-2,4-diaminobutyrate transaminase (ectB) and ectoine synthase (ectC), respectively, has been clearly characterized to be a highly conservative cluster across halophile species, allowing three-step biocatalysis to form ectoine from $L$-aspartate- $\beta$-semialdehyde (ASA), an important intermediate involved in several amino acids synthesis, such as threonine and lysine ${ }^{13-15}$. Interestingly, many halophiles also contain ectoine hydrolase encoded by ect $D$ for converting ectoine into 5-hydroxyectoine as both carbon- and nitrogen-source used under nutrient-limited conditions, which is a dominating bypass limiting the high-level intracellular accumulation of ectoine ${ }^{16,17}$.

Recent progresses have exemplified the enhanced production of ectoine by recombinant halophiles, halophilic archaea, E. coli and Corynebacterium glutamicum employing various synthetic biology strategies. Specifically, halophiles were first grown in media with a high salt concentration for sufficient ectoine synthesis, subsequently cells were quickly transferred into desalted water for effective secretion of ectoine into extracellular space, termed 'bacterial milking'18,19. Interestingly, it was reported that the deletion of TeaABC transporter in Halomonas elongata DSM 2581 led to an efficient secretion of ectoine in extracellular space during a high-cell-density fermentation ${ }^{20,21}$. Additionally, the heterologous expression of ectABC cluster from halophiles by engineered model microorganisms enabling sufficient ectoine biosynthesis has been achieved: ectABC from Chromohalobacter salexigens and Halomonas elongata DSM 2581, the most studied cluster, were overexpressed in E. coli for enhanced production of ectoine from L-aspartate and glucose ${ }^{14,22}$. Remarkably, metabolically engineered E. coli W3110 and C. glutamicum with bypass deletion, transcriptional (promoter) and translational (RBS) tuning based on high-throughput combinatory constructs and bioprocess optimizations, were successfully used to direct metabolic fluxes towards ectoine synthesis from glucose for efficient ectoine synthesis 6,23 .

Compared with current industrial biotechnology (CIB) based on model microorganisms, next generation industrial biotechnology (NGIB) based on Halomonas bluephagenesis TD01, a natural poly(3-hydroxybutyrate) (PHB) producer, has been successfully developed for achieving cost-effective production under open unsterile and continuous growth conditions ${ }^{24-27}$. PHB, a family member of microbial biopolyesters synthesized by many bacteria and archaea including E. coli, Pseudomonas putida and Cupriavidus necator et al., is a model biodegradable polymer for commercial applications ${ }^{28-32}$. H. bluephagenesis has been successfully engineered to produce cost-effective $\mathrm{PHB}^{33-35}$ from labscale to pilot-scale $(5000-\mathrm{L})$ fermentation ${ }^{27}$. It has become highly desirable to engineer $H$. bluephagenesis as a versatile chassis for productions of valuable chemicals at a competitive cost, or for co-production of PHA (polyhydroxyalkanoates, or PHB) with a small molecular chemical to increase the process economy ${ }^{36,37}$.
$H$. bluephagenesis could be a suitable host to engineer for ectoine production due to its fast growth and high PHB accumulation under high-salt conditions. So far H. bluephagenesis has been manipulated via various approaches including CRISPR/ Cas $9^{34}$, stimulus response-based flux-tuning $(\mathrm{SR}-\mathrm{FT})^{38}$, genomic integrations, inducible promoter mining 35 , and promoter engineering ${ }^{39}$. These concreted efforts enable rational strain engineering of complexity for synthesizing diverse metabolic targets, strongly expanding the capability of H. bluephagenesis as a versatile production chassis. Generally, static optimization is a widely used strategy to fine-tune the pathways involved in multi-gene regulation largely dependent on high throughput constructs and machine learning-based analysis ${ }^{40-42}$, leading to high cost of clone construction with a long R\&D cycle.

This study aims to chromosomally engineer $H$. bluephagenesis for enhanced production of ectoine from glucose using "stimulus response-based flux-tuning” method (Fig. 1). Specifically, the ectoine titer reaches 6.3 and $28 \mathrm{~g} \mathrm{~L}^{-1}$ during a 48 h growth in 500 $\mathrm{mL}$ shake flask and $28 \mathrm{~h}$ fed-batch cultivation in 7-L bioreactor, respectively, by chromosomally engineered $H$. bluephagenesis TD-ADEL-58 harboring fine-tuned ectoine synthesis pathway. At the same time, co-production of ectoine and PHB is also studied for possible economic improvement ${ }^{43-45}$, which results in $8 \mathrm{~g} \mathrm{~L}^{-1}$ ectoine and $32 \mathrm{~g} \mathrm{~L}^{-1}$ dry cell mass (DCM) containing $75 \%$ PHB after $44 \mathrm{~h}$ fed-batch fermentation.

\section{Results}

Identification of ectoine synthesis pathway. Previous studies reported that intracellular accumulation of ectoine plays the main role in hypersalinity resistance in most halophilic bacteria ${ }^{46}$, of which $H$. bluephagenesis is the one engineered as the chassis for $\mathrm{NGIB}^{47}$. As a metabolic target and compatible solute, cell growth characterization was performed to study the impact between ectoine and $\mathrm{NaCl}$ in the presence of various ectoine and $\mathrm{NaCl}$ concentrations, respectively (Fig. 2a). Interestingly, ectoine provides tolerance to $H$. bluephagenesis grown under high- and lowsalt conditions, and minor effects were observed in a 60MMG medium regardless of ectoine concentrations (Fig. 2a and Supplementary Fig. 1).

It is important to note that the de novo synthesis pathway of ectoine has been identified, it is highly conservative across species $^{6}$ (Fig. 1). Based on the genomic analysis of $H$. bluephagenesis, the ectABC gene cluster encoding the major enzymes for ectoine synthesis (Fig. 1) was annotated by several databases including RAST and KEGG. Particularly, the nucleotide blast results from NCBI database show that genes ect $A, e c t B$, and ectC of $H$. bluephagenesis shared $86.46 \%, 86.05 \%$, and $80.30 \%$ identity, respectively, compared with the ones from H. elongata DSM2581. Two strategies were employed to verify the functionality of ect $A B C$ cluster: (I) prototyping ectoine production by the start host, H. bluephagenesis TD1.0; (II) heterologous over-expressing ect $A B C$ for ectoine production by recombinant $E$. coli ${ }^{22}$.

Liquid chromatography-tandem mass spectrometry (LC-MS/ MS) was applied to assay the accumulation of ectoine by $H$. bluephagenesis TD1.0 cultivated in a $60 \mathrm{MM}$ medium supplemented with $30 \mathrm{~g} \mathrm{~L}^{-1}$ glucose in shake flask studies. Results showed that the biosynthesis ectoine shared the same retention time of mass spectra with ectoine standard, which is consistent with the secondary analysis results of LC-MS/MS (Supplementary Fig. 2). Furthermore, high performance liquid chromatography (HPLC) was used to quantify the initial production of ectoine by H. bluephagenesis TD1.0 after cell lysis, as most halophiles accumulate ectoine intracellularly for resisting high salt circumstances (Supplementary Fig. 3). As a result, a production titer of $0.63 \mathrm{~g} \mathrm{~L}^{-1}$ ectoine was obtained from $9 \mathrm{~g} \mathrm{~L}^{-1}$ DCM containing 


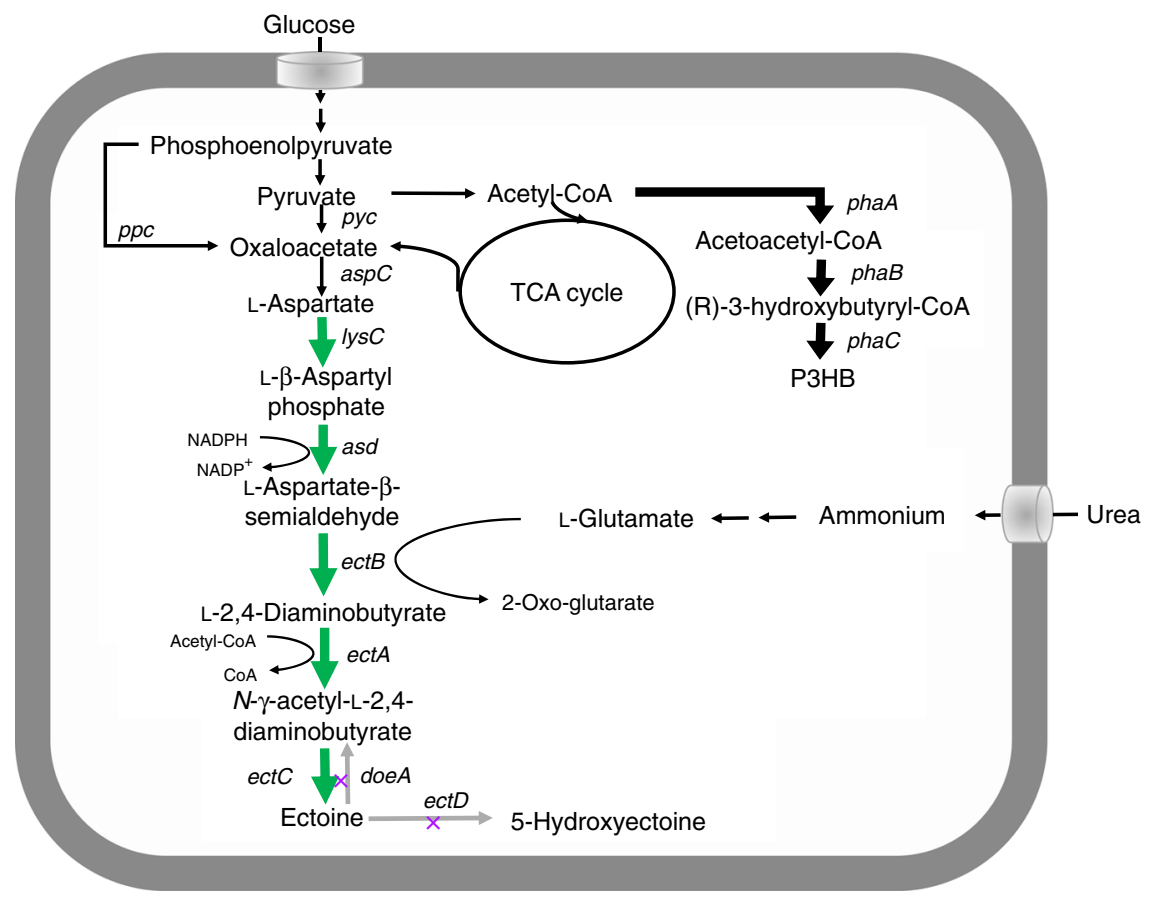

Fig. 1 Metabolic engineering for ectoine and PHB co-production by $\boldsymbol{H}$. bluephagenesis TD1.0. Endogenous metabolic fluxes of high efficiency involved in PHB synthesis are shown in black bold lines. Fine-tuned ectoine biosynthesis pathway for enhanced production is shown in green bold lines. Genes doeA and ectD putatively encoding ectoine hydrolase and ectoine hydroxylase, respectively, are deleted to reduce ectoine degradation. phaA, 3-ketothiolase; phaB, NADPH (or NADH)-dependent acetoacetyl-CoA reductase; phaC, PHA synthase; ectA, L-2,4-diaminobutyrate acetyltransferase; ectB, L-2,4diaminobutyrate transaminase; ectC, ectoine synthase; asd and lys $C$ encoding L-aspartate-semialdehyde-dehydrogenase and aspartokinase, respectively, are cloned from Corynebacterium glutamicum.

62 wt\% PHB (Fig. 2b), confirming the function of ect $A B C$ cluster in $H$. bluephagenesis. Secondly, ectABC from H. bluephagenesis was cloned into the medium-copy-number plasmid pSEVA321, for heterogenous expression by recombinant $E$. coli $\mathrm{DH} 5 a$ under the control of $\mathrm{P}_{t a c}$ promoter induced by IPTG (Fig. 2c). The titer of ectoine varied from 2 to $4 \mathrm{~g} \mathrm{~L}^{-1}$ in the presence of different IPTG concentrations for $48 \mathrm{~h}$ cultivation in the MME medium (Fig. 2d), further demonstrating the function of ectABC in $H$. bluephagenesis. Thus, more efforts would be conducted to improve the production yield of ectoine by employing various metabolic engineering approaches (Fig. 1).

Tuning the ect $A B C$ expression for enhanced ectoine synthesis. $H$. bluephagenesis was generally engineered for diverse PHA productions requiring a nitrogen limited medium, or a $60 \mathrm{MM}$ medium supplemented with $30 \mathrm{~g} \mathrm{~L}^{-1}$ glucose for shake flask cultivations. As ectoine is an aspartate derived compound heavily depending on both carbon- and nitrogen-catabolism, it is necessary to reinforce the nitrogen supply via a medium modification. Even more important, transcriptional tuning of ect $A B C$ cluster via adding $\mathrm{L}$-aspartate as precursor is significant for channeling more fluxes to ectoine, while avoiding the possibility of endogenous negative feedback control on the ect $A B C$ expression.

Based on the above understanding, a T7-like induced system was constructed to overexpress ectABC on a plasmid-based system in recombinant $H$. bluephagenesis TD1.0 in the presence of various concentrations of IPTG (Fig. 3a) ${ }^{35}$. Specifically, 100 $\mathrm{mM}$ aspartate was added in the cultural medium, namely MME (see methods), to ensure the metabolic fluxes flowing towards ectoine synthesis. As a result, the optimal transcriptional level for ect $A B C$ expression was determined after the induction of $20 \mathrm{mg} \mathrm{L}$ ${ }^{-1}$ IPTG, the maximum production titer of ectoine reached 2.38 $\mathrm{g} \mathrm{L}^{-1}$ (Fig. 3b), indicating that a medium optimization should be conducted in the presence of $20 \mathrm{mg} \mathrm{L}^{-1}$ IPTG in shake flask studies. Therefore, factorial analysis was performed to search the optimized concentration of $\mathrm{NaCl}$ in the $\mathrm{MME}$ media for enhanced ectoine production by recombinant $H$. bluephagenesis harboring ect $A B C$ expression module induced by $20 \mathrm{mg} \mathrm{L}^{-1}$ IPTG. The 60MME medium containing $60 \mathrm{~g} \mathrm{~L}^{-1} \mathrm{NaCl}$ demonstrated the highest $2.82 \mathrm{~g} \mathrm{~L}^{-1}$ ectoine when cell growth and ectoine synthesis was balanced (Fig. 3c). The ectoine titer was comparable at a higher salt concentration, but the cell growth was inhibited. In contrast, a low $\mathrm{NaCl}$ concentration also significant decreases the production titer of ectoine (Fig. 3c).

However, the use of aspartate as a nitrogen source is not suitable for cost-effective industrial ectoine production due to its high cost. Therefore, urea, a low-cost nitrogen source replaced aspartate for de novo synthesis of ectoine (Fig. 1). On the basis of ect $A B C$ flux-tuning and $\mathrm{NaCl}$ concentration modification, a urea optimized concentration was found to be at $3 \mathrm{~g} \mathrm{~L}^{-1}$, and was added to a 60MMG medium, termed $60 \mathrm{MMU}$ (see methods). This resulted in a $2.83 \mathrm{~g} \mathrm{~L}^{-1}$ ectoine produced by recombinant $H$. bluephagenesis harboring ect $A B C$ expression module induced by $20 \mathrm{mg} \mathrm{L}^{-1}$ IPTG in shake flasks (Fig. 3d), which is close to that cultured in 60MME medium. Addition of urea had a negligible impact on cell growth, yet significantly decrease the PHB content when urea exceeded $1 \mathrm{gL}^{-1}$. This info is an important hint for co-production of $\mathrm{PHB}$ and ectoine in this study.

Deletion on degradation genes for enhanced ectoine synthesis. Ectoine can be degraded in two major pathways encoded by doeA (EC 3.5.4.44) and ectD (EC 1.14.11.55) (Fig. 1) $)^{48}$, forming $\mathrm{N}$ - $\alpha$ acetyl-L-2,4-diaminobutyrate (N- $\alpha$-ADABA) and 5-hydroxyectoine, respectively. Both of these two genes were found in the genome of $H$. bluephagenesis via genomic analysis, they function 
a

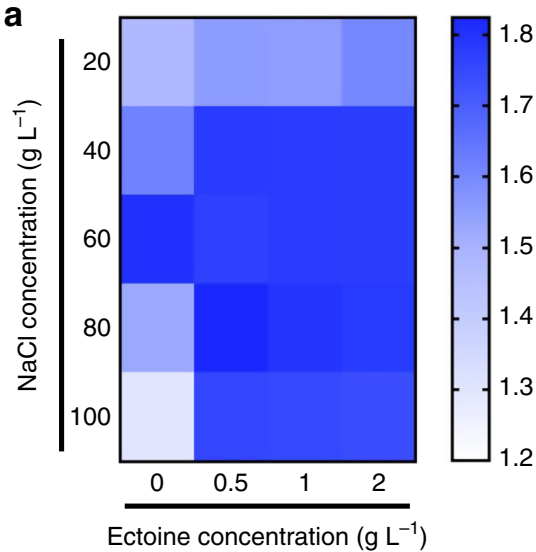

C

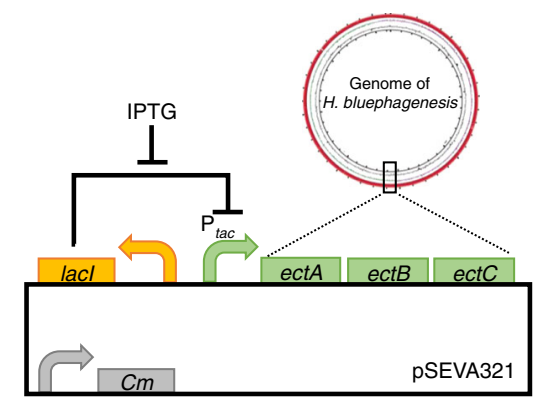

b

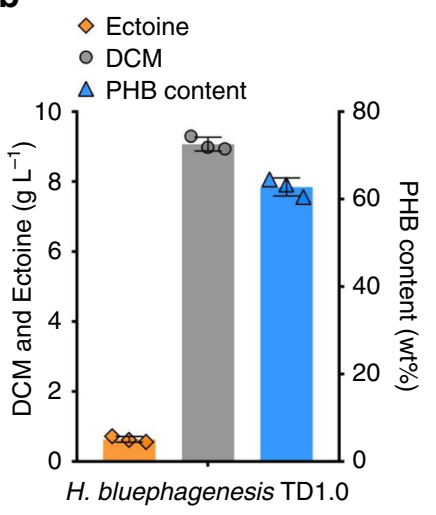

d

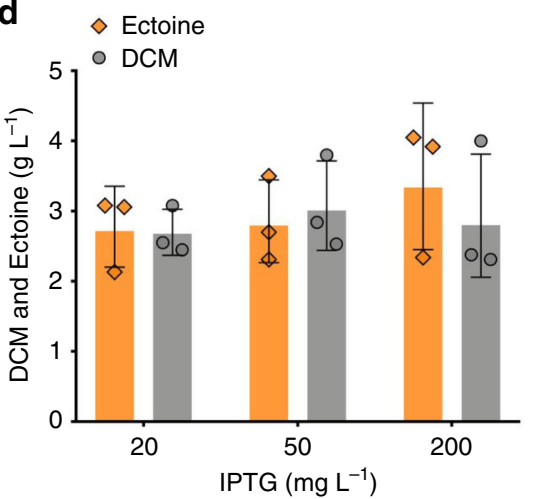

Fig. 2 Identification of the ectoine biosynthesis pathway of $\boldsymbol{H}$. bluephagenesis TD1.0. a Heatmap of cell growth in a MM medium supplemented with $10 \mathrm{~g} \mathrm{~L}^{-1}$ glucose in the presence of different $\mathrm{NaCl}$ (vertical axis) and ectoine concentrations (horizontal axis), repectively, cultured in a 96 -well plate for $24 \mathrm{~h}$. The color shade variation in blue indicates gradient value of optical density under $600 \mathrm{~nm}\left(\mathrm{OD}_{600}\right)$. b Conceptional proof of ectoine production by $H$. bluephagenesis TD1.0 cultured in a $60 \mathrm{MMG}\left(60 \mathrm{~g} \mathrm{~L}^{-1} \mathrm{NaCl}\right)$ medium used in previous studies. c Cloning the ectABC ectoine operon from $H$. bluephagenesis TD1.0 to a plasmid-based expression system pSEVA321, under the control of the tac promoter $\left(P_{\text {tac }}\right)$ inducible by IPTG. d Ectoine production by $E$. coli $\mathrm{DH} 5 \alpha$ harboring an ectABC expression module in the presence of $100 \mathrm{mM}$ sodium aspartate and three IPTG concentrations in a LB medium supplemented with $10 \mathrm{~g} \mathrm{~L}^{-1}$ glucose, respectively. Data are presented as mean values, error bars represent standard deviations (SD); $n=3$ biologically independent samples. Source data underlying (a), (b), and (d) are provided as a Source Data file.

to reduce ectoine accumulation. It is thus necessary to eliminate these two branches for blocking the degradation of ectoine by deleting $\operatorname{doe} A$ and ectD (Fig. 1). A CRISPR/Cas9 mediated gene editing tool was employed to generate single-knockout $H$. bluephagenesis TD-A $(\triangle d o e A)$, and double-knockout $H$. bluephagenesis TD-AD ( $\triangle$ doeA, $\Delta e c t D)$, respectively (Fig. $4 \mathrm{a})$. Deletion of $\operatorname{doe} A$ and ectD were confirmed based on colony PCR using two primer designs (Fig. 4a). Sequences of sgRNA designed for gene deletions were listed in Supplementary Table 2, respectively.

Subsequently, ectoine production by $H$. bluephagenesis TD01 derived strains $H$. bluephagenesis TD1.0, $H$. bluephagenesis TD$\mathrm{A}$ and $H$. bluephagenesis TD-AD harboring ectABC expression module, respectively, induced using $20 \mathrm{mg} \mathrm{L}^{-1}$ IPTG were conducted in shake flasks. Recombinant cells were cultured in 60MME (Fig. 4b) and 60MMU medium (Fig. 4c), respectively. Results showed that doeA deletion led to a $35 \%$ increase on ectoine titer, reaching up to $3.1 \mathrm{~g} \mathrm{~L}^{-1}$, and further deletion on ectD increased ectoine to only $3.2 \mathrm{~g} \mathrm{~L}^{-1}$ (Fig. $4 \mathrm{c}$ ) when cultured in the $60 \mathrm{MMU}$ medium. Interestingly, only $5 \%$ and $15 \%$ increases were observed by $H$. bluephagenesis TD-A and $H$. bluephagenesis $\mathrm{TD}-\mathrm{AD}$, respectively, when cultured in the $60 \mathrm{MME}$ medium (Fig. 4b), probably attributed to the rich aspartate dosage. Both mutants revealed that deletions of doeA and ectD had little effect on their growth (Supplementary Fig. 4), this is helpful for scale-up studies on co-production of PHB and ectoine.

Chromosomally transcriptional tuning of ectABC. Chromosome integration of target expression module(s) allows robust performance of cell growth under antibiotic-free cultivations. Therefore, a GFP-mediated transcriptional mapping approach developed for fine-tuning target genes in the previous study ${ }^{38}$ was employed to screen an appropriate constitutive promoter for chromosomal expression of the ect $A B C$ cluster (Fig. 5a). Specifically, fluorescent protein sfGFP serving as a global reporter for expression levels was applied to quantify and bridge the correlations cross various promoters and expression systems for efficiently screening the suitable promoter used to transcribe target expression modules, such as ect $A B C$, respectively ${ }^{38}$ (Supplementary Fig. 5). In this study, the T7-like induced system was characterized again by expressing $s f g p^{35}$ (Fig. 6b). Simultaneously, a porin promoter library was constructed and characterized by expressing $s f g f p$ as described in our previous study ${ }^{39}$.

Since the optimized expression strength of ect $A B C$ depends on the T7-like induced system, such as the optimized $20 \mathrm{mg} \mathrm{L}^{-1}$ IPTG induction for the T7-like system, yet IPTG is an expensive chemical not suitable for large scale applications. Thus, 
a

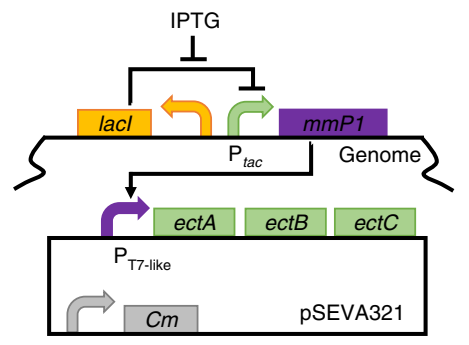

C

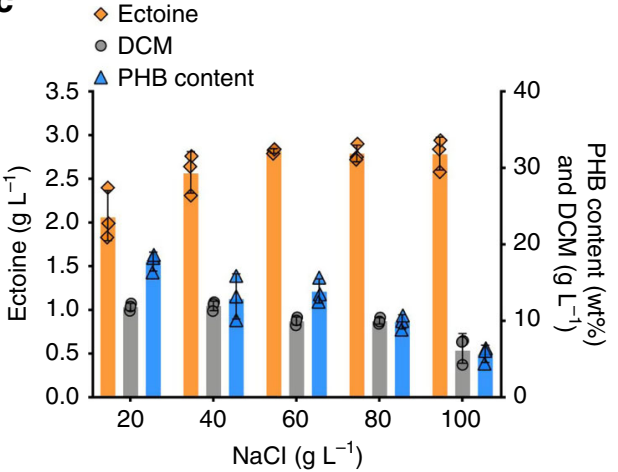

b $\diamond$ Ectoine

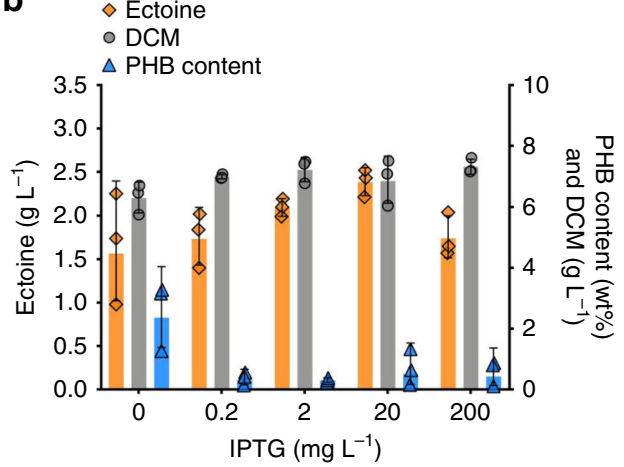

d

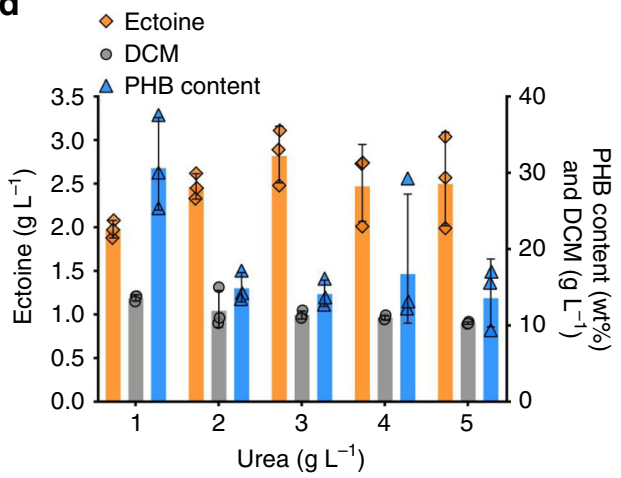

Fig. 3 Expression tuning of ect $A B C$ and medium optimization for enhanced ectoine synthesis. a Construction of ect $A B C$ expression module in a plasmidbased system in $\mathrm{H}$. bluephagenesis. A T7-like promoter $\left(\mathrm{P}_{\mathrm{T} 7 \text {-like }}\right.$ ) activated by MmP1 polymerase, which is controlled by $\mathrm{P}_{\text {tac }}$ promoter and induced by IPTG, namely, a T7-like inducible system, was employed to control the expression of ectABC operon. $\mathbf{b}$ Transcriptional tuning of ect $A B C$ expression unit for ectoine production by $\mathrm{H}$. bluephagenesis TD1.0 in the presence of various IPTG concentrations, respectively, in a 60MM medium supplemented with $100 \mathrm{mM} \mathrm{KCl}, 100 \mathrm{mM}$ sodium aspartate, $1.7 \mathrm{~g} \mathrm{~L}^{-1}$ citrate acid and $30 \mathrm{~g} \mathrm{~L}^{-1}$ glucose (short as $60 \mathrm{MME}$ ). c Growth, PHB and ectoine productions by recombinant $H$. bluephagenesis TD1.0 grown in the presence of different $\mathrm{NaCl}$ concentrations, respectively, and induced using 20 mg $L^{-1}$ IPTG based on the result of $(\mathbf{b})$. $\mathbf{d}$ Effect of urea concentration as the only nitrogen source for de novo biosynthesis of ectoine and PHA production by $H$. bluephagenesis TD1.0 grown in the $60 \mathrm{MMG}$ medium induced by $20 \mathrm{mg} \mathrm{L}^{-1}$ IPTG. Data are presented as mean values, error bars represent standard deviations (SD); $n=3$ biologically independent samples. Source data underlying (b-d) are provided as a Source Data file.

a constitutive porin promoter mutant $\mathrm{P}_{140}$, which exhibits a proximal expression level compared to the T7-like promoter induced by $20 \mathrm{mg} \mathrm{L}^{-1}$ IPTG in terms of the fluorescence intensity values (Fig. $6 \mathrm{~b}$ and Supplementary Table 3), was selected to express ectABC module on the chromosome of H. bluephagenesis TD-AD (G4 loci) (Fig. 5a), forming H. bluephagenesis TD-ADE strain. However, the ect $A B C$ controlled by a stronger promoter with a fluorescence intensity value greater than 50,000 to achieve a high expression had failed to construct in E. coli (also for conjugation purpose). Since the expression levels of target modules on plasmid pSEVA321 are reported to be 9 -fold higher than that of the chromosomal expression, which can easily lead to poor- or non-growth due to the overload of target fluxes in donor cells $(\text { E. coli } S 17-1)^{38}$. Ectoine production by ectABC fine-tuned $H$. bluephagenesis TD-ADE was carried out in shake flasks containing both 60MME and 60MMU media, forming $4.7 \mathrm{~g} \mathrm{~L}^{-1}$ and $4.3 \mathrm{~g} \mathrm{~L}^{-1}$ ectoine, respectively (Fig. $5 \mathrm{~b}$ and c). In contrast, $H$. bluephagenesis TD1.0 harboring ectABC expression module under T7-like promoter encoded on a plasmid-based expression system used as positive control termed $H$. bluephagenesis TD-AD-E, produced approximately $4 \mathrm{~g} \mathrm{~L}^{-1}$ ectoine, significantly lower than $4.6 \mathrm{~g} \mathrm{~L}^{-1}$ ectoine produced by the H. bluephagenesis TD-ADE containing the chromosome-based ectABC system under the control of porin promoter mutant $\mathrm{P}_{140}$, yet much higher than the control $H$. bluephagenesis $\mathrm{TD}-\mathrm{AD}$ strain forming only less than 2 $\mathrm{g} \mathrm{L}^{-1}$ ectoine (Fig. $5 \mathrm{~b}, \mathrm{c}$ ).
Fine-tuning of $l y s C$ and asd in genome of $H$. bluephagenesis. $H$. bluephagenesis TD-ADE containing the chromosome-based and fine-tuned ect $A B C$ system under the control of porin promoter mutant $\mathrm{P}_{140}$, has enhanced the production of ectoine (Fig. 5). It is expected that further flux-tuning of asd and $l_{y s} C$ genes encoding L-aspartate-semialdehyde-dehydrogenase and aspartokinase, respectively, could channel more metabolic fluxes toward ectoine synthesis (Fig. 1) ${ }^{16,49}$. Therefore, gene lys $C$ of Corynebacterium glutamicum and asd of $H$. bluephagenesis were overexpressed in H. bluephagenesis TD-ADE, followed by transcription tuning to obtain better performance on ectoine accumulation.

Two inducible promoters are required including T7-like promoter to modulate the expression of $l y s C$ and asd genes, simultaneously and respectively. In this case, a quorum sensingbased regulator LuxR encoded by a luxR triggering the transcription of the corresponding promoter $\mathrm{P}_{\text {lux }}$ in the presence of acyl homoserine lactone (AHL) was constructed on a plasmidbased expression system controlled by the constitutive promoter $\mathrm{P}_{\mathrm{I} 23110}{ }^{50}$, together with $s f g f p$ controlled by $\mathrm{P}_{\text {lux }}$, for the doesresponse functional characterization in $H$. bluephagenesis TDADE using flow cytometer analysis (Fig. 6a and Supplementary Fig. 6). Results exhibited 86-folds dynamic range of fluorescence intensity (FI) of sfGFP in the presence of various AHL concentrations with low basal leakiness, FI $=157$ (Supplementary Fig. 6). Subsequently, the expression module of $\mathrm{P}_{123110^{-}}-l u x R$ was integrated in G43 loci in $H$. bluephagenesis TD-ADE, forming $H$. 
a

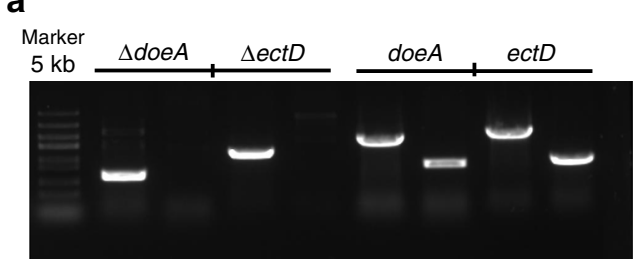

$F_{1} / R_{1} F_{2} / R_{2} F_{1} / R_{1} F_{2} / R_{2} F_{1} / R_{1} F_{2} / R_{2} F_{1} / R_{1} F_{2} / R_{2}$

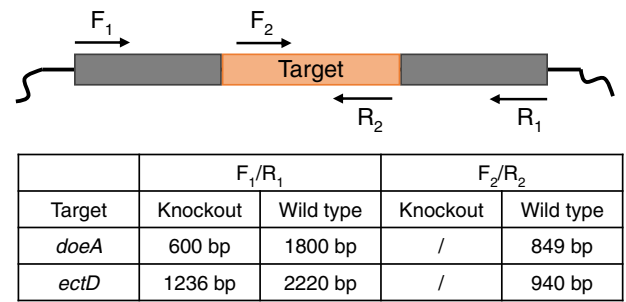

b

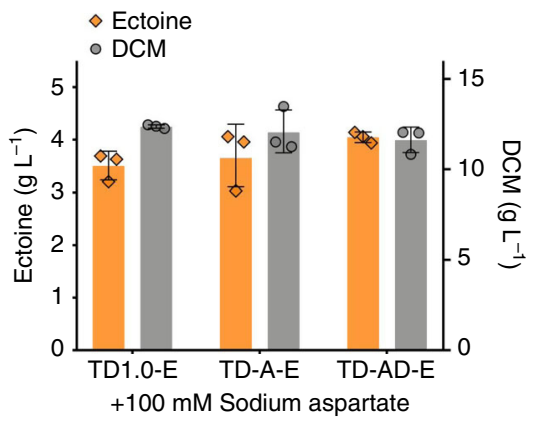

C

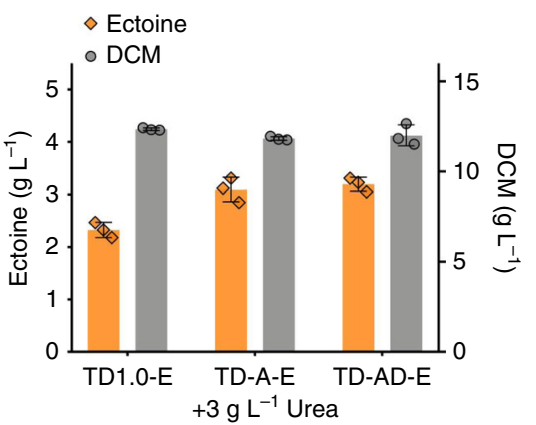

Fig. 4 Effect of deleting ectoine degradation genes doeA and/or ectD on ectoine synthesis. a Primer design and colony PCR results of doeA and ect $D$ deletion for blocking the probable degradation of ectoine. b, c Performances of ectoine accumulation by various engineered $H$. bluephagenesis TD1.0 strains, respectively, strain TD1.0: a start host, strain TD-A: single-deletion of doeA, and strain TD-AD: double-deletion of doeA and ectD genes. Strains harboring the ect $A B C$ expression module on plasmid-based systems, namely, strain TD1.0-E, TD-A-E, and TD-AD-E, respectively, induced with 20 mg L ${ }^{-1}$ IPTG. b Cells cultured in $60 \mathrm{MME}$ medium, while (c) was grown in $60 \mathrm{MMG}$ medium supplemented with extra $3 \mathrm{~g} \mathrm{~L}^{-1}$ urea (60MMU) for de novo biosynthesis of ectoine from glucose and urea only. Data are presented as mean values, error bars represent standard deviations (SD); $n=3$ biologically independent samples. Source data are provided as a Source Data file.
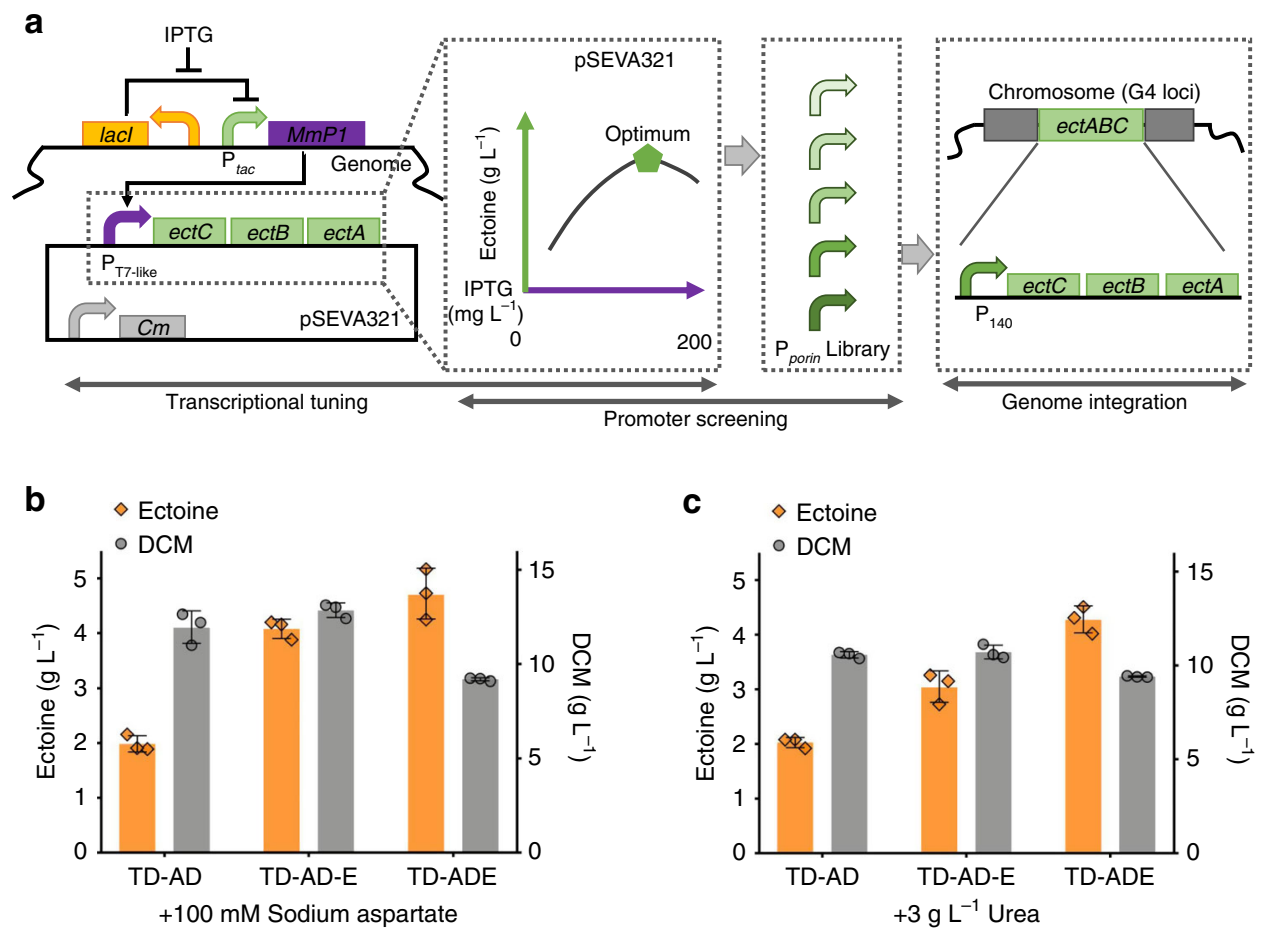

Fig. 5 Chromosomal expression of ectABC in $\boldsymbol{H}$. bluephagenesis TD1.0 for ectoine production. a Transcription-tuning design based on stimulus responsive flux-tuning approach mediated by sfGFP fluorescence intensity. Reprinted from Metabolic Engineering, 57, Jianwen Ye, Dingkai Hu, Jin Yin, Wuzhe Huang, Ruijuan Xiang, Lizhan Zhang, Xuan Wang, Jianing Han, Guo-Qiang Chen, Stimulus response-based fine-tuning of polyhydroxyalkanoate pathway in Halomonas, 85-95, Copyright (2020), with permission from Elsevier. b, c Shake flask results of ectoine production by recombinant $H$. bluephagenesis TD1.0 (TD-AD) and its derivates with ectABC expression on plasmid-based system in the presence of $20 \mathrm{mg} \mathrm{L}^{-1}$ IPTG (TD-AD-E) and chromosome-based system under the control of porin promoter mutant $\mathrm{P}_{140}$ (TD-ADE), respectively. $\mathbf{b}$ Cells cultured in a 60MME medium, while (c) were grown in the $60 \mathrm{MMU}$ medium for de novo biosynthesis of ectoine. Data are presented as mean values; error bars represent standard deviations (SD); $n=3$ biologically independent samples. Source data underlying $(\mathbf{b}, \mathbf{c})$ are provided as a Source Data file. 
a

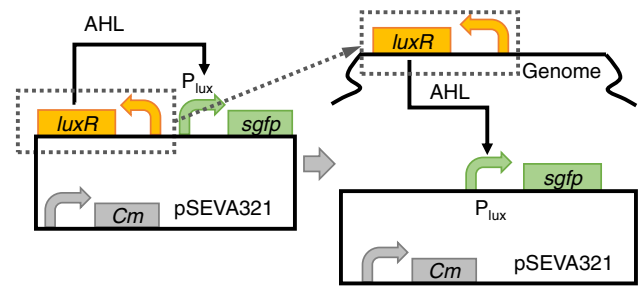

b

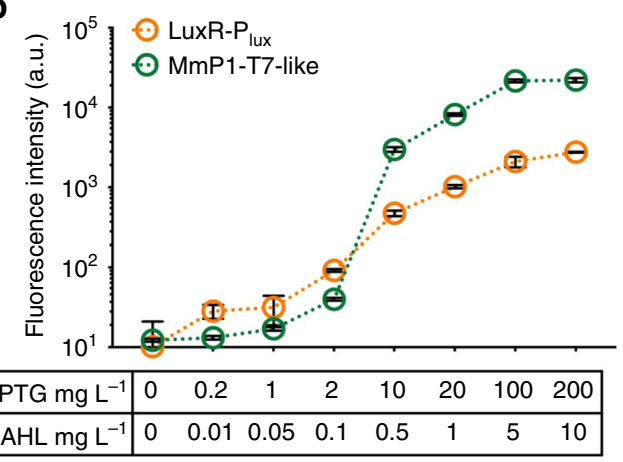

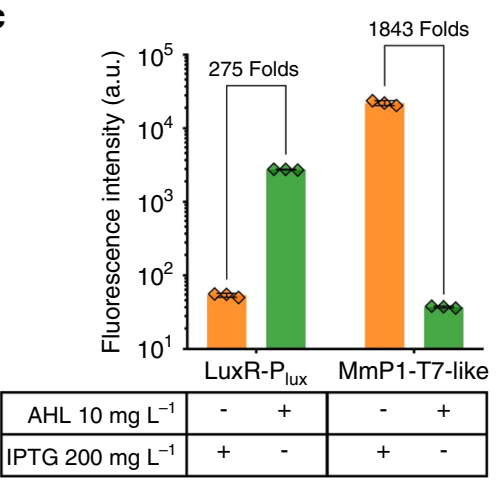

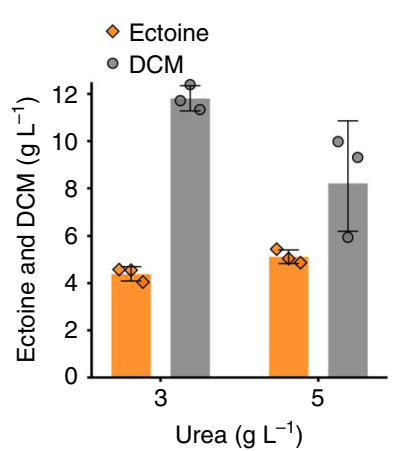

e

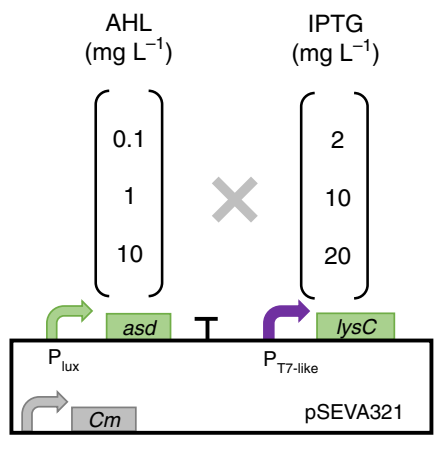

$\mathbf{f}$

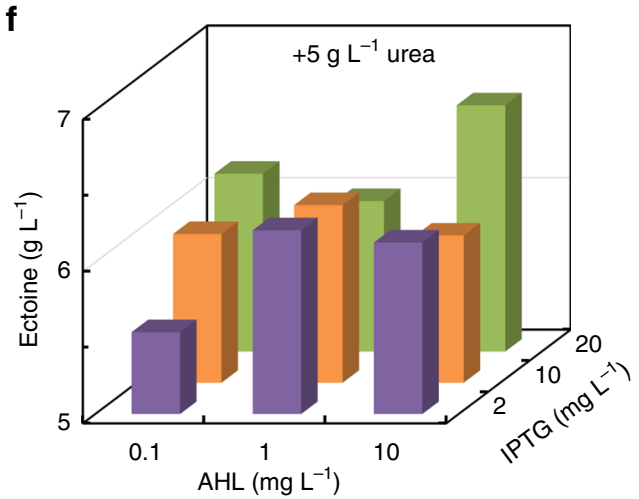

g

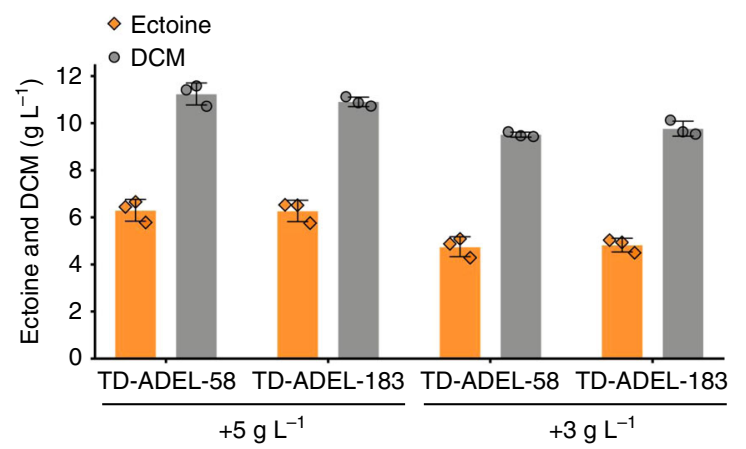

Fig. 6 Fine-tuning of asd and lysC genes based on two orthogonal inducible systems. a Construction of a luxR-AHL inducible system in $H$. bluephagenesis TD1.0 by expressing regulatory protein, LuxR, on plasmid- and chromosome-based system, respectively. b Flow cytometer characterization of the two induced systems, luxR-AHL and T7-like, respectively. c Orthogonal study of luxR-AHL and T7-like inducible systems. d Urea on cell growth and ectoine synthesis based on ectABC fine-tuned strain TD-ADE. e Design of combinatory transcription-tuning of asd and lys $C$ genes. $\mathbf{f}$ Transcriptional tuning of asd and lys $C$ genes for optimal ectoine production in 500-mL shake flask cultivation in the 60MMU medium. Data are mean values of triplicates, corresponded data points with error bars were shown in Supplementary Fig. 7 independently. $\mathbf{g}$ Shake flask studies of ectoine production by engineered $\mathrm{H}$. bluephagenesis TD1.0 strains TD-ADEL-58 and TD-ADEL-183, with fine-tuned expression modules of asd and lysC genes on chromosome based on TD-ADE in 60MMU5 and 60MMU medium, respectively. Data are presented as mean values, error bars represent standard deviations (SD); $n=3$ biologically independent samples. Source data underlying (b-d), (f), and (g) are provided as a Source Data file.

bluephagenesis TD-LuxR strain (Fig. 6a). 275- and 1843-fold dynamic ranges were obtained from H. bluephagenesis TD-LuxR under the LuxR-AHL and T7-like induced systems, respectively, the basal leakiness of chromosome-based LuxR-AHL induced system decreased to $10 \mathrm{FI}$ value (Fig. 6b). Thus, two orthogonal induced systems were successfully constructed in H. bluephagenesis enabling double-module modulation (Fig. 6c).

Ideally, expression of $l y s C$ and asd would be induced by IPTG and AHL, respectively, for enhanced ectoine production by consuming more urea. Before that, it is necessary to optimize urea concentration in the culture medium again. Addition of $5 \mathrm{~g} \mathrm{~L}^{-1}$ of urea to the 60MMG medium (namely 60MMU5) generated $17 \%$ improvement on ectoine accumulation although cell growth decreased significantly (Fig. 6d). Here, two expression modules, namely, asd and $l y s C$ controlled by $\mathrm{P}_{\mathrm{lux}}$ and $\mathrm{P}_{\mathrm{T} 7 \text {-like, respectively, }}$ were constructed on a plasmid-based system for transcriptional tuning (Fig. 6e). The induction concentrations of IPTG and AHL did have a significant effect on ectoine production. For example, an induction using $20 \mathrm{mg} \mathrm{L}^{-1}$ IPTG and $10 \mathrm{mg} \mathrm{L}^{-1}$ AHL generated the highest $6.6 \mathrm{~g} \mathrm{~L}^{-1}$ ectoine, $29 \%$ higher compared to that produced by $H$. bluephagenesis TD-ADE (Fig. 6d, f, Supplementary Fig. 7). Using the GFP-mediated transcription-tuning approach, 

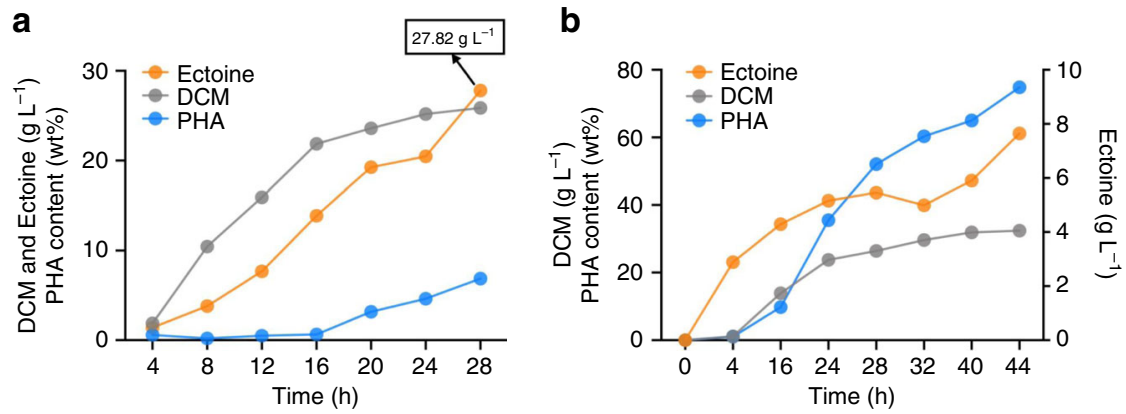

Fig. 7 Ectoine production by chromosomally engineered $\boldsymbol{H}$. bluephagenesis TD-ADEL-58. a Growth, PHB and ectoine production by chromosomally engineered $H$. bluephagenesis TD1.0 strain TD-ADEL-58 fine-tuned with the ectoine biosynthesis pathway, including three individual expression units controlled by porin promoters of various strengths, respectively. $\mathbf{b}$ Co-production of ectoine and PHB by chromosomally engineered $H$. bluephagenesis TD1.0 (TD-ADEL-58). Nitrogen source in feeding solution Feed-I/II was reduced to enhance PHB accumulation compared to (a) with poor PHB accumulation. Data are generated from one fed-batch fermentation in a 7-L bioreactor under open unsterile conditions without means and error bars. Source data are provided as a Source Data file.

porin promoter mutants $\mathrm{P}_{226}$ and $\mathrm{P}_{140}$ were selected to control the expression of $l y s C$, and $\mathrm{P}_{58}$ and $\mathrm{P}_{183}$ for asd on the chromosome of $H$. bluephagenesis TD-ADE.

However, the expression module encoding lys $C$ controlled by $\mathrm{P}_{140}$ failed to construct in the donor $E$. coli as also mentioned previously. Yet, two strains, $H$. bluephagenesis TD-ADEL-58 and TD-ADEL-183, were successfully grown for shake flask studies using the 60MMU5 medium (Fig. 6g). The two strains produced almost $6.3 \mathrm{~g} \mathrm{~L}^{-1}$ ectoine after $48 \mathrm{~h}$ cultivation in the 60MMU5 medium, over $30 \%$ higher compared to that cultured in the $60 \mathrm{MMU}$ medium. Due to the failure of $\mathrm{P}_{140^{-}}$lys $C$ construction, a weaker transcription strength of $l y s C$ controlled by $\mathrm{P}_{226}$ probably weakens the flux for L- $\beta$-aspartyl phosphate synthesis, which coupled with asd controlled by $\mathrm{P}_{183}$, it cannot bring significant increase on ectoine when grown in both 60MMU and 60MMU5 media, respectively. Thus, $H$. bluephagenesis TD-ADEL-58 became a better option for fed-batch studies in $7 \mathrm{~L}$ lab-scale bioreactor. Similarly, considering the fed-batch study for coproduction of $\mathrm{PHB}$ and ectoine, $H$. bluephagenesis TD-ADE with genomic expression of lys $C$ and asd genes were performed in a 96-well plate to investigate the effect on growth scale-up (Supplementary Fig. 4).

Fed-batch study for co-production of PHB and ectoine. $H$. bluephagenesis TD-ADEL-58 was grown for $28 \mathrm{~h}$ for ectoine production under open unsterile conditions in a $7 \mathrm{~L}$ bioreactor. $28 \mathrm{~g} \mathrm{~L}^{-1}$ ectoine were produced from this fed-batch study, which is over 4-folds higher than that of the shake flask results (Fig. 7a). The yield of ectoine on glucose and volumetric productivity reached approximately $0.21 \mathrm{~g} \mathrm{~g}^{-1}$ and $1.0 \mathrm{~g} \mathrm{~L}^{-1} \mathrm{~h}^{-1}$, respectively. Ectoine accumulation tendency was highly consistent with cell growth in terms of DCM, indicating the growth-coupled property of ectoine biosynthesis. Meanwhile, PHB accumulation was maintained at a low level during the first $16 \mathrm{~h}$ of cultivation before its increase afterward (Fig. 7a). In contrast, a fed-batch fermentation was executed for $28 \mathrm{~h}$ using $H$. bluephagenesis TD-ADE to verify the effect of fine-tuning asd and lys $C$ gene on ectoine production, leading to $17 \mathrm{~g} \mathrm{~L}^{-1}$ ectoine, which was over $30 \%$ lower than results of $H$. bluephagenesis TD-ADEL-58 (Supplementary Fig. 8).

To achieve co-production of ectoine and $\mathrm{PHB}$ by $H$. bluephagenesis TD-ADEL-58, a tailor-made feeding solution was designed to enhance $\mathrm{PHB}$ accumulation during the stationary phase using reduced nitrogen dosage in the second phase feeding solution (Feed-II). Similarly, a fed-batch fermentation was carried out in the $7 \mathrm{~L}$ bioreactor to co-produce ectoine and $\mathrm{PHB}$ in different growth phases. After a $44 \mathrm{~h}$ cultivation, the DCM reached $32 \mathrm{~g} \mathrm{~L}^{-1}$ containing $75 \% \mathrm{PHB}$, together with $8 \mathrm{~g} \mathrm{~L}^{-1}$ ectoine (Fig. 7b). It was interesting to observe that ectoine biosynthesis maintained active in the stationary phase during the fed-batch cultivation (Fig. 7b). It is expected that more bioprocess optimizations should lead to even higher ectoine and PHB production under balancing nitrogen supply in different growth phases $^{51,52}$.

\section{Discussion}

Next generation industrial biotechnology (NGIB) based on halophiles provides low-cost and convenient processing conditions for production of chemicals ${ }^{47}$. The most successful case has been exemplified by $5000 \mathrm{~L}$ scale fermentor for PHA production $^{27}$. It has been interesting to look for other examples to test possibilities of NGIB for production of non-polymer products, or even high-value added example. Ectoine, a high-value added product derived from L-aspartate synthesized by some microorganisms, has attracted increasing attentions due to its outstanding functions of stabilizing proteins and nucleic acids ${ }^{53}$. To date, many ectoine commercial applications, such as cosmetic ingredient, have been developed with high-value. However, ectoine production by wild type and engineered microorganisms yields very low productivity. Some metabolic engineering approaches have been adopted for enhanced productions of amino acids-related compounds, including ectoine ${ }^{54}$, L-threonine and L-lysine ${ }^{37}$, including new tools for strain engineering, such as dynamic and static optimization for flux enforcement ${ }^{55}$ : stimulus response-based transcriptional controls ${ }^{56,57}$ and promoter and RBS optimizations et $\mathrm{al}^{40}$. H. bluephagenesis, a high-salt tolerant halophile able to grow to a high cell density with scalability, is a promising host for ectoine synthesis because of its being a natural ectoine producer harboring the ect $A B C$ cluster in its genome. Recently, many efforts have been made to establish various genetic manipulation tools and flux-tuning approaches for improving and diversifying this NGIB chassis ${ }^{35,38}$. This study has demonstrated successful and high yield on ectoine production by chromosomally engineered $H$. bluephagenesis utilizing static optimization strategies, reaching close to $30 \mathrm{~g} \mathrm{~L}^{-1}$ ectoine from a fed-batch fermentation process (Fig. 7a).

Ectoine biosynthesis pathway of $H$. bluephagenesis was clearly identified by heterologous expression in recombinant $E$. coli, in which strong enzymatic activities of ectABC cluster were found compared with previous studies ${ }^{14,22}$ (Figs. 1, 3, and 4). Cell growth studies uncovered the enhanced resistance of cells under both low- and high-salt concentrations in the presence of ectoine, 
respectively. Furthermore, competitive ectoine degradation pathways were removed by eliminating the related genes doeA and $e c t D^{48}$, resulted in $89 \%$ improvement on ectoine production from shake flask cultivations. Two pathway branches reported by previous studies involved in L-threonine and L-lysine synthesis were retained in consideration for the benefits of ectoine coproduction with $\mathrm{PHB}^{43,51}$, a growth-dependent intracellular product, as the disruption of L-threonine and L-lysine will weaken cell growth and thus PHB accumulation.

We thus attempted to utilize transcriptional balancing strategies to boost fluxes towards ectoine synthesis. In contrast to translational regulation, transcriptional regulation could be more efficient to be carried out with minor constructs during strain engineering by onestep fine-tuning of target expression modules based on stimulus response-based flux-tuning design ${ }^{38}$. Five genes involved in ectoine synthesis pathway were grouped into three individual expression units, including ect $A B C$, lys $C$ and asd, for transcriptional fine-tuning (Figs. 1, 4). It has to be noted that quorum-sensing-based inducible system, LuxR-AHL, was firstly constructed and employed to achieve combinatory transcriptional tuning together with T7-like system in H. bluephagenesis (Figs. 5, 6). Specifically, expressions of two simple constructs, $\mathrm{p} 321-\mathrm{P}_{\mathrm{I} 23110}$-lacI- $\mathrm{P}_{\mathrm{T} 7 \text {-like-ect }}$ - $\mathrm{BC}$ and $\mathrm{p} 321-$ $\mathrm{P}_{\mathrm{T} 7 \text {-like }}-l y s C-\mathrm{P}_{\text {lux }}-a s d$, could simulate countless transcriptional combinations of target modules replacing thousands of variants construction. Finally, 9.8-folds improvement of production titer of ectoine was achieved in a shake flask study by recombinant $H$. bluephagenesis harboring fine-tuned synthesis pathway of ectoine in genome compared with $0.64 \mathrm{~g} \mathrm{~L}^{-1}$ titer yielded by the start host (Fig. 6). Subsequently, an ectoine titer of approximately $30 \mathrm{~g}$ $\mathrm{L}^{-1}$ was achieved under open unsterile fed-batch fermentation conducted in the 7-L bioreactor.

Co-production of PHB and ectoine allows harvests of intracellular bioplastic PHB and extracellular small molecular chemical ectoine, it is thus economically interesting. However, it is not sure if the two products competing for metabolic fluxes could reduce their yields. A conceptional proven study was conducted in a lab-scale fermentation. Both ectoine and PHB are growthassociated products derived from aspartate and acetyl-CoA when nitrogen source supply is abundant, approximately $28 \mathrm{~g} \mathrm{~L}^{-1}$ ectoine and $25 \mathrm{~g} \mathrm{~L}^{-1}$ DCM together with 6\% PHB were obtained, respectively, during a $28 \mathrm{~h}$ of growth (Fig. $7 \mathrm{a}$ ). In contrast, under a reduced nitrogen source, PHB accumulation was increased to $75 \%$ of the DCM in the cells producing also $8 \mathrm{~g} \mathrm{~L}^{-1}$ ectoine (Fig. 7b). In both cases, the recombinant $H$. bluephagenesis was grown to over $30 \mathrm{~g} \mathrm{~L}^{-1} \mathrm{DCM}$ that are not the highest reported by this organism ${ }^{27,58}$ (Fig. 7). Further efforts to increase the recombinants' growth density should lead to much improved ectoine and PHB productions.

Etoine as a representative product of $\mathrm{L}$-aspartate compound family with a high value, should be able to produce at a competitive cost after fine-tuning of every single gene of ect $A B C$ cluster employing SR-FT approach by developing more suitable inducible systems ${ }^{59}$. Although static optimization is a widely used strategy for enhanced production of metabolic targets, recent progresses focused on bifunctional dynamic control provide a strong hint for improving the production of ectoine. Since co-production of PHB and ectoine seems to be competing for flux, the efficiency to activate target pathways dynamically for PHB and ectoine must be considered during the fermentation processes ${ }^{60,61}$. In addition, the bioprocessing optimization, such as feeding solution optimization ${ }^{62}$ and cascaded bioprocessing ${ }^{52}$, is a promising strategy to obtain simultaneously enhanced production yields for ectoine and PHB. Additionally, attempts of enhancing export system ${ }^{48}$ and developing amino acids responsive regulators ${ }^{57}$ could also be applied to achieve further improvement on ectoine and PHB production under cost-effective bio-manufactural process based on the NGIB chassis.

In conclusion, the halophile NGIB chassis can be effectively used for effective production of ectoine. Engineering the ectoine pathways improved ectoine production effectively. Co-production of intracellular PHA and non-polymer chemicals is possible using the halophilic chassis.

\section{Methods}

Strains culture and chemicals. $\mathrm{LB}$ medium is composed of $\left(\mathrm{g} \mathrm{L}^{-1}\right): 10$ trypton, 5 yeast extract and $10 \mathrm{NaCl}$. 60LB medium is derived from $\mathrm{LB}$ medium supplemented with $60 \mathrm{~g} \mathrm{~L}^{-1} \mathrm{NaCl}$. 60MMG medium consists of $\left(\mathrm{g} \mathrm{L}^{-1}\right)$ : glucose $30, \mathrm{NaCl}$ 60 , yeast extract $1,\left(\mathrm{NH}_{4}\right)_{2} \mathrm{SO}_{4} 0.25, \mathrm{MgSO}_{4} 0.2, \mathrm{Na}_{2} \mathrm{HPO}_{4} \cdot 12 \mathrm{H}_{2} \mathrm{O} 9.65, \mathrm{KH}_{2} \mathrm{PO}_{4}$ 1.5 , trace element solution I $10 \mathrm{~mL} \mathrm{~L}^{-1}$ and trace element solution II $1 \mathrm{~mL} \mathrm{~L}^{-1}$. The composition of trace element solution I consists of $\left(\mathrm{g} \mathrm{L}^{-1}\right)$ : $\mathrm{Fe}(\mathrm{III})-\mathrm{NH}_{4}$-citrate 5 , $\mathrm{CaCl}_{2} 2, \mathrm{HCl} 1 \mathrm{M}$. The trace element solution II $\left(\mathrm{mg} \mathrm{L}^{-1}\right)$ consists of: $\mathrm{ZnSO}_{4} \cdot 7 \mathrm{H}_{2} \mathrm{O}$ $100, \mathrm{MnCl}_{2} \cdot 4 \mathrm{H}_{2} \mathrm{O} 30, \mathrm{H}_{3} \mathrm{BO}_{3} 300, \mathrm{CoCl}_{2} \cdot 6 \mathrm{H}_{2} \mathrm{O} 200, \mathrm{CuSO}_{4} \cdot 5 \mathrm{H}_{2} \mathrm{O} 10, \mathrm{NiCl}_{2} \cdot 6 \mathrm{H}_{2} \mathrm{O}$ 20 , and $\mathrm{NaMoO}_{4} \cdot 2 \mathrm{H}_{2} \mathrm{O} 30$. 60MME is derived from 60MMG medium supplemented with $100 \mathrm{mM}$ sodium aspartate with additional $100 \mathrm{mM} \mathrm{KCl}$, and $1.7 \mathrm{~g} \mathrm{~L}^{-1}$ citric acid. Alternatively, $100 \mathrm{mM}$ sodium aspartate in 60MME medium was replaced by 3 and $5 \mathrm{~g} \mathrm{~L}^{-1}$, respectively, to form 60MMU and 60MMU5 media for de novo synthesis of ectoine for shake flask studies. Ectoine of analytical purity purchased from Sigma-Aldrich (St. Louis, MO, USA) was used as standard for HPLC analysis for fermentative ectoine quantitation.

$H$. bluephagenesis TD1.0 derived from H. bluephagenesis TD01, isolated from Aydingol Lake of China, was used to engineer for production of ectoine in this study. Escherichia coli S17-1pir, the donor strain for conjugation, was cultivated in LB medium. Seed preparation of $H$. bluephagenesis and its derivates in this study were cultured in 60LB medium. Co-production of ectoine and PHA in shake flasks by recombinant $H$. bluephagenesis TD were cultivated in 60MMG, 60MME, 60MMU and 60MMU5 media, respectively.

All the derivates from $H$. bluephagenesis TD1.0, wild type H. bluephagenesis TD integrated with expression module of MmP1 RNA polymerase induced by IPTG, were listed in Supplementary Table 1. Firstly, doeA and ectD gene encoding bypass (ectoine degradation) enzymes were deleted, forming H. bluephagenesis TD-AD strain. Then, expression modules including ect $A B C$, lys $C$ and asd cluster were integrated into the chromosome of $H$. bluephagenesis one-by-one at G4, G7 and G43 locus, respectively, forming H. bluephagenesis strains TD-ADE, TD-ADEL-58, and TD-ADEL-183.

Plasmids construction. Plasmids were constructed to carry functional gene expressions encoded in vector pSEVA32163. All the constructs were assembled by Gibson Assembly kit, and conjugated into $H$. bluephagenesis derivates. DNA extraction was carried out by using Tiangen Plasmid Extraction kit (Shanghai, China) and Omega Gel Purification kit (USA). DNA oligonucleotides were synthesized by Zixi Biotechnology (Beijing, China). Genetic design and sequence reading were performed by Snap Gene (v2.3.2).

All the plasmids were constructed by Gibson Assembly kit. For gel purification the Omega gel purification kit was used, and the Tiangen plasmid extraction kit was used to obtain the plasmids. All the plasmids used in this study and genes required fine-tuning to obtain enhanced ectoine production were listed in Supplementary Table 1. The sequences of sgRNA used for genome editing in the study were listed in Supplementary Table 2. CRSPR-based genomic editing approach was employed to construct various recombinant strains by gene knockout and gene integration ${ }^{34}$. Firstly, plasmid pQ08 containing Cas9 expression module was transformed into recombinant $H$. bluephagenesis by conjugation. Followed by the conjugation of constructs derived from pSEVA241 vector carrying gRNA and homologous arms ( $500 \mathrm{bp}$ ), positive colonies were screened by PCR test and confirmed by DNA sequencing after the discard of plasmids by passaging the cell cultures in $60 \mathrm{LB}$ medium without antibiotics for 3-5 generations.

Conjugation. Firstly, plasmids were constructed by E. coli DH5a, subsequently transformed into E. coli S17-1, the donor strain for conjugation. Then, donor cells harboring target constructs were cultivated for $12-14 \mathrm{~h}$ in $150-\mathrm{mL}$ shake flasks containing $20 \mathrm{~mL} \mathrm{LB}$ medium, and recipient cells, recombinant $H$. bluephagenesis, were cultivated in 60LB medium overnight. Both of the donor and recipient cells were centrifugated and harvested under $4^{\circ} \mathrm{C}$, washed with a fresh medium and recovering to the same volume that sampled. Subsequently, cells were mixed (1:1 volume) and dropped on antibiotic-free 20 -LB agar plates, followed by $6-8 \mathrm{~h}$ incubation at $37^{\circ} \mathrm{C}$ for conjugation. Finally, conjugated cells from the lawn were suspended with 60LB medium and spread on 60LB agar plates containing relevant antibiotics for $36 \mathrm{~h}$ incubation to obtain positive colonies.

Characterization of LuxR-AHL and T7-like inducible systems. LuxR-AHL inducible system was first constructed on plasmid-based expression system in $H$. bluephagenesis TD1.0 harboring plasmid $\mathrm{p} 321-\mathrm{P}_{\mathrm{J} 23110}-l u x R-\mathrm{P}_{\text {lux }}-s f g f p$ to characterize the performance in the presence of various AHL (OC6, from Sigma) 
concentrations, respectively. Then, luxR expression module was integrated into the chromosome of H. bluephagenesis TD-ADE at G43 loci, forming TD-LuxR strain, followed by the characterization of LuxR-AHL and T7-like induced systems in $H$. bluephagenesis TD-LuxR strain harboring plasmids, $\mathrm{p} 321-\mathrm{P}_{\mathrm{lux}}-\mathrm{s} f f \mathrm{f} p$ and $\mathrm{p} 321-\mathrm{P}_{\mathrm{T} 7}$ like $s f g f p$ after conjugation. Cells carrying target expression vessels were plated on $60 \mathrm{LB}$ agar plate added with relevant antibiotics for $16-20 \mathrm{~h}$ of incubation at $37^{\circ} \mathrm{C}$. Subsequently, single colony was inoculated into $1 \mathrm{~mL}$ 60LB medium for $12 \mathrm{~h}$ cultivation in 96-deep-well plate covered with sealing films (BF-400-S; ThermoShaker, Aosheng, $37^{\circ} \mathrm{C}, 1000 \mathrm{rpm}$ ). Then, $5 \mu \mathrm{L}$ cell culture was inoculated into a new plate supplemented with appropriate antibiotics in presence of a spectrum of inducer. After $12 \mathrm{~h}$ incubation, cell cultures were diluted 250-folds using phosphate-buffered saline solution (PBS) for flow cytometry analysis.

Positive fluorescent cells were recorded by flow cytometer (LSRFortessa4, BD bioscience, USA) at the rate of $0.5 \mu \mathrm{L} \mathrm{s}^{-1}$ for $20 \mathrm{~s}$ with at least 30,000 cell captured in total of each sample, and the start host, $H$. bluephagenesis TD1.0, was used as the negative control groups to modify the fluorescence levels of experimental groups. Intracellular sfGFP protein was excited under $488 \mathrm{~nm}$, and cells were captured on the signal channels of FITC, FSC (forward scatter) and SSC (side scatter). The FlowJo (v7.6) software was used to process the raw data for obtaining mean value of fluorescence intensity and percentage of positive cells.

Cell growth study. To study the effects of ectoine and $\mathrm{NaCl}$ on cell growth, single colony of recombinant $H$. bluephagenesis TD1.0 was first picked as an inoculum for a $12 \mathrm{~h}$ incubation in $1 \mathrm{~mL} 60 \mathrm{LB}$ medium in a 96-deep-well plate (Corning/USA, $1 \mathrm{~mL} 60 \mathrm{LB}$ medium) at $37^{\circ} \mathrm{C}$. Subsequently, seed cultures were diluted 200 -folds with fresh MM medium supplemented with $20 \mathrm{~g} \mathrm{~L}^{-1}$ in the presence of various ectoine and $\mathrm{NaCl}$ concentrations in a new 96-well plate (flat bottom, $250 \mu \mathrm{L}$ medium per well) for online recording of optical density at $600 \mathrm{~nm}\left(\mathrm{OD}_{600}\right)$ for $48 \mathrm{~h}$.

To characterize cell growth of recombinant $H$. bluephagenesis in this study, chromosomally engineered strains were pre-cultured in a 60-LB medium in the 96deep-well plate for $12 \mathrm{~h}$ by picking a single colony as an inoculum. Then, seed cultures were diluted 200 -folds with a fresh 60LB medium in a new 96-well plate (flat bottom, $250 \mu \mathrm{L}$ medium per well) for online monitoring of optical density at $600 \mathrm{~nm}\left(\mathrm{OD}_{600}\right)$ for $24 \mathrm{~h}$. The cell growth characterization was carried out using a micro-plate reader (Varioskan Flash, Thermo Scientific).

Shake flask and fed-batch studies. For shake flask studies, recombinant $H$. bluephagenesis harboring target expression modules on plasmid- or chromosomebased systems were pre-cultured overnight to obtain seed cultures $\left(\mathrm{OD}_{600}\right.$ reached $2.5 \pm 0.2$ ) from a single colony, respectively. Subsequently, $2.5 \mathrm{~mL}$ seed culture were inoculated into $50 \mathrm{~mL} 60 \mathrm{MM}$ medium supplemented with $30 \mathrm{~g} \mathrm{~L}^{-1}$ glucose and nitrogen source (urea or sodium aspartate) whenever necessary. $25 \mathrm{\mu g} \mathrm{mL}^{-1}$ chloramphenicol was added to the medium grown with $H$. bluephagenesis harboring the plasmid-carried expression systems. Cell cultures were harvested for dry cell mass (DCM), ectoine and PHB content assays after $48 \mathrm{~h}$ cultivation. Additionally, functional verification of gene cluster ectABC from $H$. bluephagenesis was performed using the same procedure of recombinant $H$. bluephagenesis grown in LB medium and recombinant $E$. coli $\mathrm{DH} 5 \alpha$ harboring plasmid $\mathrm{p} 321-\mathrm{J}_{23110}-\mathrm{lacI}-$ Ptac-ectABC.

For fed-batch studies, cells were grown overnight in 60LB medium in $500 \mathrm{~mL}$ shake flasks to obtain $300 \mathrm{~mL}$ seed cultures $\left(\mathrm{OD}_{600}\right.$ reached $\left.2.5 \pm 0.2\right)$ as inoculums in a fed-batch study conducted in a 7-L bioreactor (NBS Bioflo3000, New Brunswick, USA). A $3 \mathrm{~L} 60 \mathrm{MM}$ medium supplemented with $3 \mathrm{~g} \mathrm{~L}^{-1} \mathrm{CO}\left(\mathrm{NH}_{2}\right)_{2}$, $10 \mathrm{~g} \mathrm{~L}^{-1}$ yeast extract and $20 \mathrm{~g} \mathrm{~L}^{-1}$ glucose was used as an initial medium for fermentation. During the fermentation process, dissolved oxygen (DO\%) was maintained at $\sim 30 \%$ of air saturation by injecting air with a maximum flow rate of 1 VVM (air volume per culture volume per min) by coupling the agitation of less than $800 \mathrm{rpm}$. $\mathrm{pH}$ was adjusted using $5 \mathrm{M} \mathrm{NaOH}$ solution. Feed solution of the first phase (Feed-I) is composed of $200 \mathrm{~g}$ glucose, $8 \mathrm{~g}$ yeast extract and $32 \mathrm{~g}$ urea $(24 \mathrm{~g}$ of urea for co-production purpose). For ectoine production, the second phase feeding solution (Feed-II) consists of $200 \mathrm{~g}$ glucose, $4 \mathrm{~g}$ yeast extract and $28 \mathrm{~g}$ urea, while fed nutrients composed of $200 \mathrm{~g}$ glucose, $4 \mathrm{~g}$ yeast extract and $8 \mathrm{~g}$ urea for coproduction of ectoine and PHB. And Feed-III containing only $800 \mathrm{~g} \mathrm{~L}^{-1}$ glucose was fed when Feed-II ran out during the fermentation for co-production of ectoine and PHB. Feed solutions were fed to maintain the residual glucose at 6-10 g L (measured by SANNUO medical glucometer, China). All batches of 7-L fermentation were conducted under non-sterilized open condition at $37^{\circ} \mathrm{C}$. Then, cells sampled of every time point were harvested for cell mass, ectoine and PHB assays.

Assays of dry cell mass and PHB content. First, cells were harvested from $30 \mathrm{~mL}$ fermentative broth during a fed-batch study or at the end of the shake flask study via centrifugation at $12,200 \mathrm{~g}$ (CR 21GIII, HITACHI, Japan) for $15 \mathrm{~min}$, subsequently suspended and washed twice with distilled water. A dry cell mass was calculated by measuring the mass of harvested cells after $24 \mathrm{~h}$ of lyophilization under $-60^{\circ} \mathrm{C}$ and $100 \mathrm{~Pa}$. For PHB content assays, $30-40 \mathrm{mg}$ lyophilized cells in powder form were sampled for methanolysis under $100^{\circ} \mathrm{C}$ for $3.5 \mathrm{~h}$ supplemented with $2 \mathrm{~mL}$ chloroform and $2 \mathrm{~mL}$ methanolysis solution ( $85 \mathrm{wt} \%$ methanol, $15 \mathrm{wt} \%$
$\mathrm{H}_{2} \mathrm{SO}_{4}$, and $1 \mathrm{~g} \mathrm{~L}^{-1}$ benzoic acid) mixture ${ }^{48}$. Then, $1 \mathrm{~mL}$ water was added to the methanolysis solution after cooling down to room temperature $\left(25^{\circ} \mathrm{C}\right)$ for extraction and phase separation. $1 \mathrm{~mL}$ of the heavy (low) phase was sampled for PHB content calculation using GC (gas chromatography, GC-2014, SHIMADZU, Japan) analysis. 15 and $20 \mathrm{mg}$ pure PHB (3HB standard from Sigma-Aldrich) were used as standards.

Ectoine assays. Five millimeters of fermentative broth from $30 \mathrm{~mL}$ cell cultures during fed-batch study or at the end of shake flasks were diluted 10-folds (50-folds for the fed-batch study) in a $50 \mathrm{~mL}$ tube. Subsequently, cells were disrupted by a high pressure homogenizer and centrifugated at $12,200 \mathrm{~g}$ for $10 \mathrm{~min}$. Then, the supernatants were filtrated once by $0.22 \mu \mathrm{m}$ filter membrane for ectoine quantification using high-performance liquid chromatography (HPLC, SHIMADZU, Japan) equipped with a C18 column based on acetonitrile/water mixture (70:30, v/ v) at a flow rate of $1 \mathrm{~mL}$ per min as the mobile phase. UV (ultraviolet) detector $(210 \mathrm{~nm})$ was used for ectoine detection. The HPLC-MS analysis was carried out using OrbiTrap Q-Exactive LC-MS (Thermo Scientific) under the same conditions.

Reporting summary. Further information on research design is available in the Nature Research Reporting Summary linked to this article.

\section{Data availability}

The datasets generated and analyzed during the current study are available from the corresponding authors upon request. A reporting summary for this Article is available as a Supplementary Information file. The datasets generated and analyzed during the current study are available from the corresponding author upon request. Plasmids sequences are available in Supplementary Data 1-13. Source data are provided with this paper.

Received: 14 February 2020; Accepted: 16 June 2020; Published online: 03 July 2020

\section{References}

1. Galinski, E. A., Pfeiffer, H. P. \& Truper, H. G. 1,4,5,6-Tetrahydro-2-methyl-4pyrimidinecarboxylic acid: a novel cyclic amino acid from halophilic phototrophic bacteria of the genus. Ectothiorhodospira. Eur. J. Biochem. 149, 135-139 (1985).

2. Arakawa, T. \& Timasheff, S. N. The stabilization of proteins by osmolytes. Biophys. J. 47, 411-414 (1985)

3. Auton, M., Rosgen, J., Sinev, M., Holthauzen, L. M. \& Bolen, D. W. Osmolyte effects on protein stability and solubility: a balancing act between backbone and side-chains. Biophys. Chem. 159, 90-99 (2011).

4. Bolen, D. W. \& Baskakov, I. V. The osmophobic effect: natural selection of a thermodynamic force in protein folding. J. Mol. Biol. 310, 955-963 (2001)

5. Bremer, E. \& Kramer, R. Responses of microorganisms to osmotic stress. Annu. Rev. Microbiol. 73, 313-334 (2019).

6. Czech, L., Poehl, S., Hub, P., Stoveken, N. \& Bremer, E. Tinkering with osmotically controlled transcription allows enhanced production and excretion of ectoine and hydroxyectoine from a microbial cell factory. Appl. Environ. Microbiol. 84, e01772-17 (2018).

7. Lentzen, G. \& Schwarz, T. Extremolytes: natural compounds from extremophiles for versatile applications. Appl. Microbiol. Biotechnol. 72, 623-634 (2006).

8. Pastor, J. M. et al. Ectoines in cell stress protection: uses and biotechnological production. Biotechnol. Adv. 28, 782-801 (2010).

9. Graf, R., Anzali, S., Buenger, J., Pfluecker, F. \& Driller, H. The multifunctional role of ectoine as a natural cell protectant. Clin. Dermatol. 26, 326-333 (2008).

10. Bownik, A. \& Stepniewska, Z. Ectoine as a promising protective agent in humans and animals. Arh. Hig. Rada. Toksikol. 67, 260-265 (2016).

11. Salar-Garcia, M. J. et al. Understanding the interplay of carbon and nitrogen supply for ectoines production and metabolic overflow in high density cultures of Chromohalobacter salexigens. Microb. Cell Fact. 16, 23 (2017).

12. Czech, L., et al. Role of the extremolytes ectoine and hydroxyectoine as stress protectants and nutrients: genetics, phylogenomics, biochemistry, and structural analysis. Genes (Basel) 9, 177 (2018).

13. Goller, K., Ofer, A. \& Galinski, E. A. Construction and characterization of an $\mathrm{NaCl}$-sensitive mutant of Halomonas elongata impaired in ectoine biosynthesis. FEMS Microbiol. Lett. 161, 293-300 (1998).

14. Ning, Y. et al. Pathway construction and metabolic engineering for fermentative production of ectoine in Escherichia coli. Metab. Eng. 36, 10-18 (2016).

15. Richter, A. A. et al. Biosynthesis of the stress-protectant and chemical chaperon ectoine: biochemistry of the transaminase EctB. Front Microbiol. 10, 2811 (2019). 
16. Lee, K. H., Park, J. H., Kim, T. Y., Kim, H. U. \& Lee, S. Y. Systems metabolic engineering of Escherichia coli for L-threonine production. Mol. Syst. Biol. 3, 149 (2007).

17. Lo, C. C., Bonner, C. A., Xie, G., D’Souza, M. \& Jensen, R. A. Cohesion group approach for evolutionary analysis of aspartokinase, an enzyme that feeds a branched network of many biochemical pathways. Microbiol. Mol. Biol. Rev. 73, 594-651 (2009).

18. Sauer, T. \& Galinski, E. A. Bacterial milking: a novel bioprocess for production of compatible solutes. Biotechnol. Bioeng. 59, 128 (1998).

19. Schubert, T., Maskow, T., Benndorf, D., Harms, H. \& Breuer, U. Continuous synthesis and excretion of the compatible solute ectoine by a transgenic, nonhalophilic bacterium. Appl Environ. Microbiol. 73, 3343-3347 (2007).

20. Grammann, K., Volke, A. \& Kunte, H. J. New type of osmoregulated solute transporter identified in halophilic members of the bacteria domain: TRAP transporter TeaABC mediates uptake of ectoine and hydroxyectoine in Halomonas elongata DSM 2581(T). J. Bacteriol. 184, 3078-3085 (2002).

21. Schwibbert, K. et al. A blueprint of ectoine metabolism from the genome of the industrial producer Halomonas elongata DSM 2581 T. Environ. Microbiol. 13, 1973-1994 (2011).

22. He, Y. Z. et al. High production of ectoine from aspartate and glycerol by use of whole-cell biocatalysis in recombinant Escherichia coli. Microb. Cell. Fact. 14, 55 (2015).

23. Wendisch, V. F. Microbial production of amino acid-related compounds. Adv. Biochem. Eng. Biotechnol. 159, 255-269 (2017).

24. Fu, X. Z. et al. Development of Halomonas TD01 as a host for open production of chemicals. Metab. Eng. 23, 78-91 (2014)

25. Li, T. et al. Semirational approach for ultrahigh poly(3-hydroxybutyrate) accumulation in Escherichia coli by combining one-step library construction and high-throughput screening. ACS Synth. Biol. 5, 1308-1317 (2016).

26. Tan, D., Xue, Y. S., Aibaidula, G. \& Chen, G. Q. Unsterile and continuous production of polyhydroxybutyrate by Halomonas TD01. Bioresour. Technol. 102, 8130-8136 (2011)

27. Ye, J. et al. Pilot Scale-up of poly(3-hydroxybutyrate-co-4-hydroxybutyrate) production by Halomonas bluephagenesis via cell growth adapted optimization process. Biotechnol. J. 13, e1800074 (2018).

28. Chen, G. Q. A microbial polyhydroxyalkanoates (PHA) based bio- and materials industry. Chem. Soc. Rev. 38, 2434-2446 (2009).

29. Chen, G. Q. \& Hajnal, I. The 'PHAome'. Trends Biotechnol. 33, 559-564 (2015).

30. Koller, M., Marsalek, L., de Sousa Dias, M. M. \& Braunegg, G. Producing microbial polyhydroxyalkanoate (PHA) biopolyesters in a sustainable manner. N. Biotechnol. 37, 24-38 (2017).

31. Park, S. J., Ahn, W. S., Green, P. R. \& Lee, S. Y. Production of poly(3hydroxybutyrate-co-3-hydroxyhexanoate) by metabolically engineered Escherichia coli strains. Biomacromolecules 2, 248-254 (2001).

32. Tyo, K. E., Fischer, C. R., Simeon, F. \& Stephanopoulos, G. Analysis of polyhydroxybutyrate flux limitations by systematic genetic and metabolic perturbations. Metab. Eng. 12, 187-195 (2010).

33. Ling, C. et al. Engineering $\mathrm{NADH} / \mathrm{NAD}(+)$ ratio in Halomonas bluephagenesis for enhanced production of polyhydroxyalkanoates (PHA) Metab. Eng. 49, 275-286 (2018).

34. Qin, Q. et al. CRISPR/Cas9 editing genome of extremophile Halomonas spp. Metab. Eng. 47, 219-229 (2018).

35. Zhao, H. et al. Novel T7-like expression systems used for Halomonas. Metab. Eng. 39, 128-140 (2017).

36. Yin, J., Chen, J. C., Wu, Q. \& Chen, G. Q. Halophiles, coming stars for industrial biotechnology. Biotechnol. Adv. 33, 1433-1442 (2015).

37. $\mathrm{Li}, \mathrm{Y}$. et al. Current status on metabolic engineering for the production of $\mathrm{L}$ aspartate family amino acids and derivatives. Bioresour. Technol. 245, 1588-1602 (2017).

38. Ye, J. et al. Stimulus response-based fine-tuning of polyhydroxyalkanoate pathway in Halomonas. Metab. Eng. 57, 85-95 (2020).

39. Shen, R. et al. Promoter engineering for enhanced $\mathrm{P}(3 \mathrm{HB}-\mathrm{co}-4 \mathrm{HB})$ production by Halomonas bluephagenesis. ACS Synth. Biol. 7, 1897-1906 (2018).

40. Young, E. M. et al. Iterative algorithm-guided design of massive strain libraries, applied to itaconic acid production in yeast. Metab. Eng. 48, 33-43 (2018).

41. Zelezniak, A. et al. Machine learning predicts the yeast metabolome from the quantitative proteome of kinase knockouts. Cell Syst. 7, 269-283 e266 (2018).

42. Zhou, Y. et al. MiYA, an efficient machine-learning workflow in conjunction with the YeastFab assembly strategy for combinatorial optimization of heterologous metabolic pathways in Saccharomyces cerevisiae. Metab. Eng. 47, 294-302 (2018).

43. Guzman, H., Van-Thuoc, D., Martin, J., Hatti-Kaul, R. \& Quillaguaman, J. A process for the production of ectoine and poly(3-hydroxybutyrate) by Halomonas boliviensis. Appl. Microbiol. Biotechnol. 84, 1069-1077 (2009).

44. Chen, Q., Zhang, L., Li, X., Liu, S. \& Li, D. Poly- $\beta$-hydroxybutyrate/ectoine coproduction by ectoine-excreting strain Halomonas salina. Process Biochem. 49, 33-37 (2014).
45. Arumugam, A. \& Furhana Shereen, M. Bioconversion of calophyllum inophyllum oilcake for intensification of rhamnolipid and polyhydroxyalkanoates co-production by Enterobacter aerogenes. Bioresour. Technol. 296, 122321 (2020).

46. Kempf, B. \& Bremer, E. Uptake and synthesis of compatible solutes as microbial stress responses to high-osmolality environments. Arch. Microbiol. 170, 319-330 (1998).

47. Chen, G. Q. \& Jiang, X. R. Next generation industrial biotechnology based on extremophilic bacteria. Curr. Opin. Biotechnol. 50, 94-100 (2018).

48. Zhao, Q. et al. High ectoine production by an engineered Halomonas hydrothermalis $\mathrm{Y} 2$ in a reduced salinity medium. Microb. Cell Fact. 18, 184 (2019).

49. Becker, J. et al. Systems metabolic engineering of Corynebacterium glutamicum for production of the chemical chaperone ectoine. Microb. Cell Fact. 12, 110 (2013).

50. Zeng, W. et al. Rational design of an ultrasensitive quorum-sensing switch. ACS Synth. Biol. 6, 1445-1452 (2017).

51. Cai, L. et al. Comparative genomics study of polyhydroxyalkanoates (PHA) and ectoine relevant genes from Halomonas sp. TD01 revealed extensive horizontal gene transfer events and co-evolutionary relationships. Microb. Cell Fact. 10, 88 (2011).

52. Fallet, C., Rohe, P. \& Franco-Lara, E. Process optimization of the integrated synthesis and secretion of ectoine and hydroxyectoine under hyper/hypoosmotic stress. Biotechnol. Bioeng. 107, 124-133 (2010).

53. Schroter, M. A. et al. Ectoine protects DNA from damage by ionizing radiation. Sci. Rep. 7, 15272 (2017).

54. Perez-Garcia, F., Ziert, C., Risse, J. M. \& Wendisch, V. F. Improved fermentative production of the compatible solute ectoine by Corynebacterium glutamicum from glucose and alternative carbon sources. J. Biotechnol. 258, 59-68 (2017).

55. Holtz, W. J. \& Keasling, J. D. Engineering static and dynamic control of synthetic pathways. Cell 140, 19-23 (2010).

56. Zhang, F., Carothers, J. M. \& Keasling, J. D. Design of a dynamic sensorregulator system for production of chemicals and fuels derived from fatty acids. Nat. Biotechnol. 30, 354-359 (2012).

57. Fang, M. et al. Intermediate-sensor assisted push-pull strategy and its application in heterologous deoxyviolacein production in Escherichia coli. Metab. Eng. 33, 41-51 (2016).

58. Ren, Y., Ling, C., Hajnal, I., Wu, Q. \& Chen, G. Q. Construction of Halomonas bluephagenesis capable of high cell density growth for efficient PHA production. Appl. Microbiol. Biotechnol. 102, 4499-4510 (2018).

59. Meyer, A. J., Segall-Shapiro, T. H., Glassey, E., Zhang, J. \& Voigt, C. A. Escherichia coli "Marionette" strains with 12 highly optimized small-molecule sensors. Nat. Chem. Biol. 15, 196-204 (2019).

60. Gupta, A., Reizman, I. M., Reisch, C. R. \& Prather, K. L. Dynamic regulation of metabolic flux in engineered bacteria using a pathway-independent quorum-sensing circuit. Nat. Biotechnol. 35, 273-279 (2017).

61. Dinh, C. V. \& Prather, K. L. J. Development of an autonomous and bifunctional quorum-sensing circuit for metabolic flux control in engineered Escherichia coli. Proc. Natl Acad. Sci. 116, 25562-25568 (2019).

62. Chen, W. C. et al. Production and characterization of ectoine using a moderately halophilic strain Halomonas salina BCRC17875. J. Biosci. Bioeng 125, 578-584 (2018).

63. Silva-Rocha, R. et al. The Standard European Vector Architecture (SEVA): a coherent platform for the analysis and deployment of complex prokaryotic phenotypes. Nucleic Acids Res. 41, D666-D675 (2013).

\section{Acknowledgements}

This research was financially supported by grants from the Ministry of Science and Technology of China (Grant No. 2018YFA0900200), National Natural Science Foundation of China (Grant Nos. 31961133019, 21761132013, 31870859), China Postdoctoral Science Foundation (Grant No. 2018M640117) and Center of Life Sciences of TsinghuaPeking University. The SEVA plasmids were kindly donated by Professor Victor de Lorenzo from the National Centre for Biotechnology in Madrid, Spain.

\section{Author contributions}

G.Q.C. and J.Y. proposed the idea; G.Q.C. and J.Y. designed and supervised the experiments; H.M. performed the HPLC-MS/MS experiments and medium optimization; Y.Z. performed the genome editing experiments and the tests; H.M., Y.Z., W.H., and L.Z., performed the fermentor experiments; H.M., Y.Z., and F. W. performed all other experiments; H.M., Y.Z., J.Y., and G.Q.C analyzed the data and wrote the manuscript; all authors have read and approved the final manuscript.

\section{Competing interests}

The authors declare no competing interests. 


\section{Additional information}

Supplementary information is available for this paper at https://doi.org/10.1038/s41467020-17223-3.

Correspondence and requests for materials should be addressed to J.Y. or G.-Q.C.

Peer review information Nature Communications thanks Martin Koller, and the other, anonymous, reviewer(s) for their contribution to the peer review of this work. Peer reviewer reports are available.

Reprints and permission information is available at http://www.nature.com/reprints

Publisher's note Springer Nature remains neutral with regard to jurisdictional claims in published maps and institutional affiliations. (c) (i) Open Access This article is licensed under a Creative Commons Attribution 4.0 International License, which permits use, sharing, adaptation, distribution and reproduction in any medium or format, as long as you give appropriate credit to the original author(s) and the source, provide a link to the Creative Commons license, and indicate if changes were made. The images or other third party material in this article are included in the article's Creative Commons license, unless indicated otherwise in a credit line to the material. If material is not included in the article's Creative Commons license and your intended use is not permitted by statutory regulation or exceeds the permitted use, you will need to obtain permission directly from the copyright holder. To view a copy of this license, visit http://creativecommons.org/ licenses/by/4.0/.

(C) The Author(s) 2020 